\title{
Dynamics of a hydrogenated silica xerogel: A neutron scattering study
}

\author{
A. Fontana, ${ }^{1,2, *}$ L. Orsingher, ${ }^{1,2}$ F. Rossi, ${ }^{1,2}$ and U. Buchenau ${ }^{3}$ \\ ${ }^{1}$ Dipartimento di Fisica, Università di Trento, Via Sommarive 14, 38050 Povo, Trento, Italy \\ ${ }^{2}$ INFM CRS-SOFT, clo Università di Roma "La Sapienza," 00185 Roma, Italy \\ ${ }^{3}$ Institut für Festkörperforschung, Forschungszentrum Jülich, 52425 Jülich, Germany
}

(Received 28 April 2006; revised manuscript received 9 October 2006; published 15 November 2006)

\begin{abstract}
Neutron scattering measurements of a hydrogenated vitreous silica (or xerogel) sample reveal an unexpectedly high mean-square displacement of the silanol hydrogen atoms at 150 and $300 \mathrm{~K}$. At $150 \mathrm{~K}$, the spectrum mimics the boson peak of vitreous silica. The finding suggests a large local displacement of the boson peak modes at the breaking points of the continuous random network.
\end{abstract}

DOI: 10.1103/PhysRevB.74.172304

PACS number(s): 63.50.+x, 78.35.+c, 61.12.Ex

The confinement of water in porous silica is a wellstudied subject. ${ }^{1-4}$ At low hydration, the water molecules form a layer of interfacial water on the pore surface, which is rendered hydrophilic by the silanol terminal $\mathrm{OH}$ bonds. While much is known about the water dynamics of the interfacial layer, there is practically no information on the dynamics of the silanol bonds. The present paper supplies such information from an inelastic neutron scattering experiment on a xerogel sample, studied earlier by some of us with Raman scattering and low-temperature heat capacity measurements. ${ }^{5}$

The silica gels were prepared by hydrolysis of mixtures of triethoxylane and tetraethoxilane dissolved in ethanol. Details concerning the preparation of xerogels are given elsewhere. ${ }^{6}$

The silica-xerogel sample with nominal stoichiometric formula $\mathrm{Si}_{8} \mathrm{O}_{15} \mathrm{H}_{2}$ was annealed for $24 \mathrm{~h}$ at $500{ }^{\circ} \mathrm{C}$. After annealing, the density of the sample was $1940 \mathrm{~kg} / \mathrm{m}^{3}$. In the $\mathrm{SiO}_{2}$ matrix, pores of $4 \mathrm{~m}^{2} / \mathrm{g}$ (determined by adsorbing $\mathrm{N}_{2}$ at $77 \mathrm{~K})$ are present. In these pores, one has to reckon with additional hydrogen atoms, partially as $\mathrm{OH}$ silanol bonds on the inner surface and partially as interfacial water inside closed pores.

Measurements at 150 and $300 \mathrm{~K}$ were done on the cold neutron time-of-flight spectrometer IN6 at the High Flux Reactor of the Institute Laue-Langevin at Grenoble. The wavelength of the incoming neutrons was $5.1 \AA$ with a resolution of $70 \mu \mathrm{eV}$.

In the standard data treatment, the empty container was subtracted and the measurement was normalized to vanadium and corrected for the wavelength-dependent detector sensitivity. The multiple scattering (a small contribution because the sample was a weak scatterer) was corrected assuming isotropic multiple scattering.

The elastic scattering from the sample is taken to be a sum of an incoherent hydrogen part and a coherent $a-\mathrm{SiO}_{2}$ part. The latter is assumed to be the same as in vitreous silica. $^{7}$ We fit the elastic scattering of the hydrogenated sample with the function

$$
I_{\text {elast }}(Q)=f_{S} S_{s}(Q) e^{-\alpha Q^{2}}+f_{\mathrm{H}} e^{-2 \alpha_{\mathrm{H}} Q^{2}},
$$

with the three fit parameters $f_{s}, f_{\mathrm{H}}$, and $\alpha_{\mathrm{H}}$, where $s$ and $\mathrm{H}$ stand for silica and hydrogen, respectively. $\alpha=0.005 \AA^{2}$ is taken from Ref. 8 and the $S_{s}(Q)$ is taken from a diffraction measurement of pure silica. ${ }^{7}$ The result is shown in Fig. 1. The fit is restricted to $Q$ values above $0.6 \AA^{-1}$, because one has to reckon with small-angle scattering from pores.

According to this fit, the scattering from the hydrogen atoms is about five times stronger than the one from $\mathrm{SiO}_{2}$. Taking the scattering cross sections $2.18 \mathrm{~b}$ for $\mathrm{Si}, 4.24 \mathrm{~b}$ for $\mathrm{O}$, and $81 \mathrm{~b}$ for $\mathrm{H}$, one calculates from the data of Fig. $1 \mathrm{a}$ ratio of hydrogen to $\mathrm{SiO}_{2}$ of 0.66 . This means an average composition formula $a-\mathrm{SiO}_{2} \mathrm{H}_{0.66}$, i.e., one hydrogen per every three oxygen atoms, about twice as much hydrogen as in the nominal composition. This implies that the heat treatment has not totally removed the confined water in the xerogel pores. We conclude that, in the case of a treated sample, about half of the hydrogen atoms are situated in the pores, either as $\mathrm{OH}$ surface bonds or as interfacial water.

The Debye-Waller coefficients give mean-square hydrogen displacements $\alpha_{\mathrm{H}}=0.036 \AA^{2}$ at $150 \mathrm{~K}$ and $\alpha_{\mathrm{H}}=0.14 \AA^{2}$ at $300 \mathrm{~K}$.

To determine the vibrational density of states, one needs to know whether the inelastic signal comes only from the hydrogen or both from the hydrogen and the $\mathrm{SiO}_{2}$ component. In order to decide that, one compares the data at $10 \mathrm{meV}$ and $150 \mathrm{~K}$ to data from pure vitreous silica in the same frequency window. This is done in Fig. 2.

Figure 2 shows within experimental error a purely incoherent inelastic signal in the hydrogenated sample. Pure

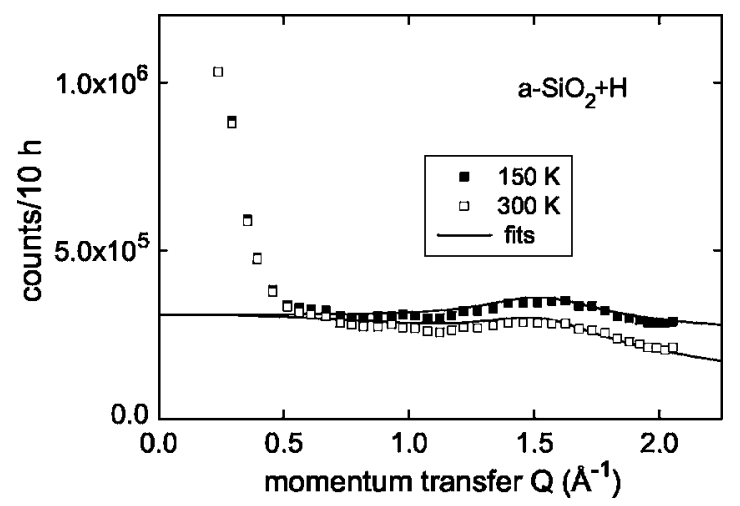

FIG. 1. Elastic scattering from the hydrogenated silica sample at $150 \mathrm{~K}$ (full squares) and $300 \mathrm{~K}$ (open squares). The continuous lines represent the fitting function (1). 


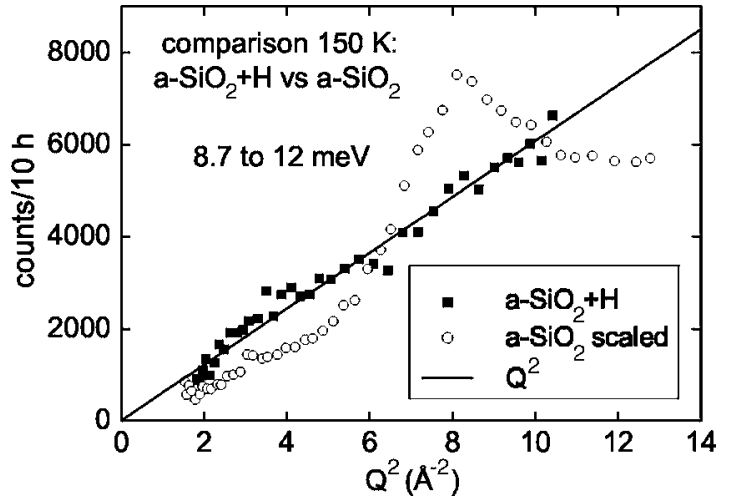

FIG. 2. Coherent inelastic signal of pure silica (open circles, scaled) compared to the one of the hydrogenated sample (full squares). The continuous line represents the $Q^{2}$ dependence.

vitreous silica shows a coherent inelastic signal oscillating around a $Q^{2}$ behavior. ${ }^{9}$ From the analysis of the elastic line, we know that one-fifth of the signal of the hydrogenated sample stems from $\mathrm{SiO}_{2}$. However, since there is no visible sign of the coherent oscillations in the hydrogenated sample data, we conclude that the inelastic signal may be attributed essentially to the hydrogen and that the inelastic $\mathrm{SiO}_{2}$ contribution is small.

This conclusion is in fact consistent with the findings on the elastic line described above. At $150 \mathrm{~K}$, the hydrogen atoms in the hydrogenated sample have a seven times larger mean square displacement than the silicon and oxygen atoms in vitreous silica. Therefore, they dominate the inelastic signal not only by their larger scattering, but also by their larger partial vibrational density of states. This view is further supported by measurements on porous silica samples ${ }^{10}$ and opals. ${ }^{11}$

To determine the vibrational density of states as a function of the energy transfer $E=\hbar \omega$, we fit the inelastic intensity with the two parameters $f_{\text {inel }}(E)$ and $\alpha_{\text {inel }}(E)$ in the expression

$$
I_{\text {inel }}(E, Q)=f_{\text {inel }}(E) Q^{2} e^{-\alpha(E) Q^{2}} .
$$

In this scheme, ${ }^{12}$ one can remove the multiphonon influence by an appropriate iteration procedure, calculating the vibrational density of states from the prefactor $f_{\text {inel }}(E)$ and then calculating the expected effective Debye-Waller coefficients $\alpha_{\text {inel }}(E)$ in the Gaussian approximation. This scheme worked quite well for the $150 \mathrm{~K}$ data. In the $300 \mathrm{~K}$ data, we found strong indications for non-Gaussianity (even stronger than that found in polymers ${ }^{12,13}$ ). The $300 \mathrm{~K}$ nonGaussianity is consistent with a bimodal distribution centered around $0.3 \AA^{2}$ (for interfacial water ${ }^{4}$ ) and $0.08 \AA^{2}$ for the silanol protons.

From the effective vibrational density of states, one can calculate the Debye-Waller factor. The values of the DebyeWaller exponent determined in this way are $\alpha_{\mathrm{H}}=0.036 \AA^{2}$ at $150 \mathrm{~K}$ and $\alpha_{\mathrm{H}}=0.17 \AA^{2}$ at $300 \mathrm{~K}$. They agree within the error bars with the values determined from the elastic line in the first section, thus showing the consistency of the whole analysis.

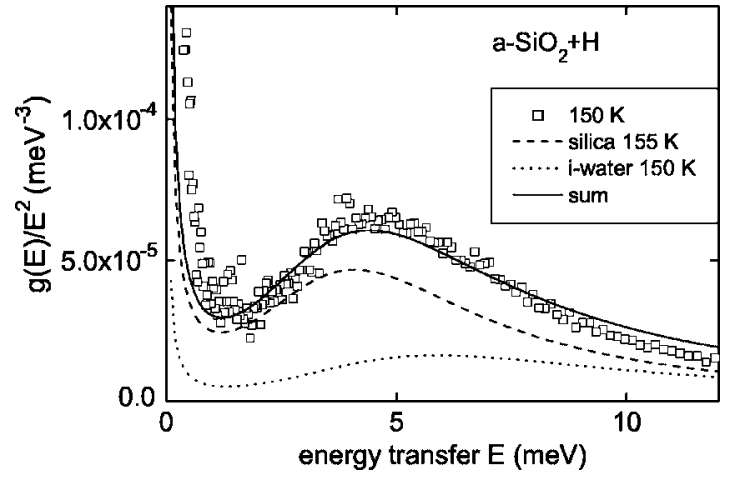

FIG. 3. Density of states of hydrogen in hydrogenated silica at $150 \mathrm{~K}$ (open squares). These data are decomposed into the (scaled) density of states of pure silica (Ref. 9) at $155 \mathrm{~K}$ (dashed line) and the (scaled) density of states of interfacial water at $150 \mathrm{~K}$ (Ref. 4) (dotted line).

The elastic $300 \mathrm{~K}$ value $\alpha_{\mathrm{H}}=0.14 \AA^{2}$ allows us to estimate the interfacial water fraction. Zanotti et al. ${ }^{4}$ report a translational $\alpha_{\mathrm{H}}$ of $0.21 \AA^{2}$ at $300 \mathrm{~K}$. Together with the rotational $\alpha_{\mathrm{H}}$ of $0.09 \AA^{2}$, this supplies the $0.3 \AA^{2}$ mentioned above. At $150 \mathrm{~K}$, both our measurement and the interfacial water data have $\alpha_{\mathrm{H}}=0.036 \AA^{2}$. The silanol $\mathrm{OH}$ is expected to show reasonably harmonic temperature behavior, yielding $\alpha_{\mathrm{H}}=0.072 \AA^{2}$ at $300 \mathrm{~K}$.

The interfacial water proton fraction $x$ is then calculated from the equation

$$
0.14=0.3 x+0.072(1-x)
$$

yielding a fraction of $30 \%$ of interfacial water protons.

This value is further supported by the $150 \mathrm{~K}$ boson peak data in Fig. 3. The measured density of states is reasonably well reproduced by a sum of silica and interfacial water spectra, weighing the silica spectrum ${ }^{9}$ with $70 \%$ and the interfacial water spectrum ${ }^{4}$ with $30 \%$. Note that this decomposition is able to explain satisfactorily the shift of the measured boson peak to a higher value $(\sim 4.7 \mathrm{meV})$ than the pure silica one $(\sim 4 \mathrm{meV})$.

We conclude that the hydrogen atoms in the hydrogenated silica sample mimic the motions in pure silica-but with a much higher amplitude. The factor of 7 in the mean square displacement at $150 \mathrm{~K}$ translates into an amplitude factor of 2.6, a rather drastic effect.

How can one understand this very large mean square displacement? From the heat capacity measurements, ${ }^{5,14}$ we know that the density of states of the xerogel is approximately equal to the one of pure silica, so the number of vibrational modes must be the same. Obviously, the amplitude of the protons in an $\mathrm{OH}$ bound to the silica network, either within the network or as silanol on a pore surface, must be considerably larger than the amplitudes of the oxygen and the silicon atoms.

The first explanation which comes to mind is to attribute this larger amplitude to the tetrahedra libration character ${ }^{9}$ of the boson peak modes in silica. However, the structure of $\mathrm{OH}$ within silica ${ }^{15}$ or as silanol on silica surfaces ${ }^{16}$ is sufficiently well known. The Si-O-H angle is of the order of 
$113^{\circ}$, so even for a pure tetrahedra rotation one does not expect more than a factor of 1.3 between hydrogen and oxygen amplitude, a factor of 2 less than the one observed here.

A better explanation is in terms of the concept of defects in the continuous random network of an ideal glass. ${ }^{17}$ Vitreous silica is an ideal glass in Philipps' ${ }^{17,18}$ counting scheme for strong constraints. For this assignment, both the $\mathrm{Si}-\mathrm{O}$ distance and the O-Si-O bond bending are taken to be a strong constraint; the $\mathrm{Si}-\mathrm{O}-\mathrm{Si}$ angle is assumed to be completely flexible. Then one constrains nine degrees of freedom per molecule, leaving no structural flexibility to the continuous random network. The hydrogens form $\mathrm{OH}$ bonds, thus introducing an unconnected corner into the corner-connected $\mathrm{SiO}_{4}$-tetrahedra random network. It is obvious that such an interruption creates a rather mobile corner. Such a defect is expected to have unusual dynamical properties. In fact, silica samples with a small $\mathrm{OH}$ content showed additional tunneling states, the properties of which were clearly different from the intrinsic ones. ${ }^{19-22}$

With the composition $a-\mathrm{SiO}_{2} \mathrm{H}_{0.46}$ determined here (after subtraction of the interfacial water), about one-fifth of the corner connections of the network are replaced with mobile $\mathrm{OH}$ groups. Many of these $\mathrm{OH}$ groups will be sitting at inner surfaces. But even those sitting in the random matrix are free from constraining covalent bonds. Relatively free to move, they mirror and magnify the vibrations of the random silica network.

In conclusion, in this work we measured the vibrational density of states in hydrogenated xerogels by means of neutron measurements. We were able to decompose the scattering into a smaller interfacial water component and a larger fraction of protons bonded to the silica network. The $g(\omega)$ of the latter is very similar to that of amorphous silica in the boson peak frequency range, ${ }^{5,14,23}$ but the amplitude of the protons in these modes is more than twice as large as the one of the network atoms.

The authors are grateful to R. Campostrini of the University of Trento for preparing and characterizing the samples. This work was supported by INFM CRS-SOFT.
*Electronic address: afontana@ science.unitn.it

${ }^{1}$ T. Takamuku, M. Yamagami, H. Wakita, Y. Masuda, and T. Yamaguchi, J. Phys. Chem. B 101, 5730 (1997).

${ }^{2}$ F. Bruni, M. A. Ricci, and A. K. Soper, J. Chem. Phys. 109, 1478 (1998).

${ }^{3}$ P. Gallo and M. Rovere, J. Phys.: Condens. Matter 15, 7625 (2003).

${ }^{4}$ J.-M. Zanotti, M.-C. Bellissent-Funel, and S. H. Chen, Europhys. Lett. 71, 91 (2005).

${ }^{5}$ A. Fontana, F. Rossi, G. Carini, G. D’Angelo, G. Tripodo, and A. Bartolotta, Phys. Rev. Lett. 78, 1078 (1997).

${ }^{6}$ A. Fontana, E. Moser, F. Rossi, R. Campostrini, and G. Carturan, J. Non-Cryst. Solids 212, 292 (1997).

${ }^{7}$ P. A. V. Johnson, A. C. Wright, and R. N. Sinclair, J. Non-Cryst. Solids 58, 109 (1983).

${ }^{8}$ A. Wischnewski, Ph.D. thesis, University of Düsseldorf, 1998.

${ }^{9}$ A. Wischnewski, U. Buchenau, A. J. Dianoux, W. A. Kamitakahara, and J. L. Zarestky, Phys. Rev. B 57, 2663 (1998).

${ }^{10}$ U. Buchenau, M. Monkenbusch, G. Reichenauer, and B. Frick, J. Non-Cryst. Solids 145, 121 (1992).

${ }^{11}$ I. Sosnowska, U. Buchenau, G. Reichenauer, H. Graetsch, K. Ibel, and B. Frick, Physica B 234-236, 455 (1997).
${ }^{12}$ U. Buchenau, C. Pecharroman, R. Zorn, and B. Frick, Phys. Rev. Lett. 77, 659 (1996).

${ }^{13}$ J. Teixeira, M.-C. Bellissent-Funel, S. H. Chen, and A. J. Dianoux, Phys. Rev. A 31, 1913 (1985).

${ }^{14}$ A. Fontana, R. Dell'Anna, M. Montagna, F. Rossi, G. Viliani, G. Ruocco, M. Sampoli, U. Buchenau, and A. Wischnewski, Europhys. Lett. 47, 56 (1999).

${ }^{15}$ V. G. Plotnichenko, V. O. Sokolov, and E. M. Dianov, J. NonCryst. Solids 261, 186 (2000).

${ }^{16}$ J. B. Peri, J. Phys. Chem. 70, 2937 (1966).

${ }^{17}$ J. C. Phillips, J. Non-Cryst. Solids 34, 153 (1979).

${ }^{18}$ M. F. Thorpe, J. Non-Cryst. Solids 57, 355 (1983).

${ }^{19}$ M. v. Schickfus and S. Hunklinger, J. Phys. C 9, L439 (1976).

${ }^{20}$ B. Golding, M. v. Schickfus, S. Hunklinger, and K. Dransfeld, Phys. Rev. Lett. 43, 1817 (1979).

${ }^{21}$ W. A. Phillips, Philos. Mag. B 43, 747 (1981).

${ }^{22}$ J. Reinisch and A. Heuer, Phys. Rev. Lett. 95, 155502 (2005); A. Heuer (unpublished).

${ }^{23}$ S. Caponi, G. Carini, G. D'Angelo, A. Fontana, O. Pilla, F. Rossi, F. Terki, G. Tripodo, and T. Woignier, Phys. Rev. B 70, 214204 (2004). 